\title{
A 21-year-old female patient with dyspnoea, chest pain and pleural thickening on chest radiographs
}

\section{Case report}

A 21 -year-old female patient was referred to the current authors' hospital in January 2002, with dyspnoea and pleural chest pain.

The patient had been using bromocriptine to treat a hypophyseal adenoma, which was diagnosed in October 1999, after an investigation into the cause of her menstrual irregularity. The initial dose was $5 \mathrm{mg} \cdot \mathrm{day}^{?}$.

During the follow-up period, in May 2000, the patient was admitted once to hospital and all her laboratory findings and chest radiographs were normal.

On follow-up, the patient developed loss of sight. A palliative trans-sphenoidal resection was performed in June 2000. However, 1 month later, the patient's serum prolactin level was elevated, and bromocriptine was started again at a dose of 30 mg.day'. By December 2000, the dosage was increased to $60 \mathrm{mg} \cdot$ day $^{1}$.

On admission, documentation of patient history showed no previous smoking or alcohol consumption, and, apart from her prolactinoma, she appeared healthy. She had no history of asbestos exposure. Physical examination established a decrease in right lung respiratory sounds. No other abnormalities were identified. Erythrocyte sedimentation rate (ESR) was $18 \mathrm{~mm}$. Chest radiography was performed and the results are shown in figure 1.

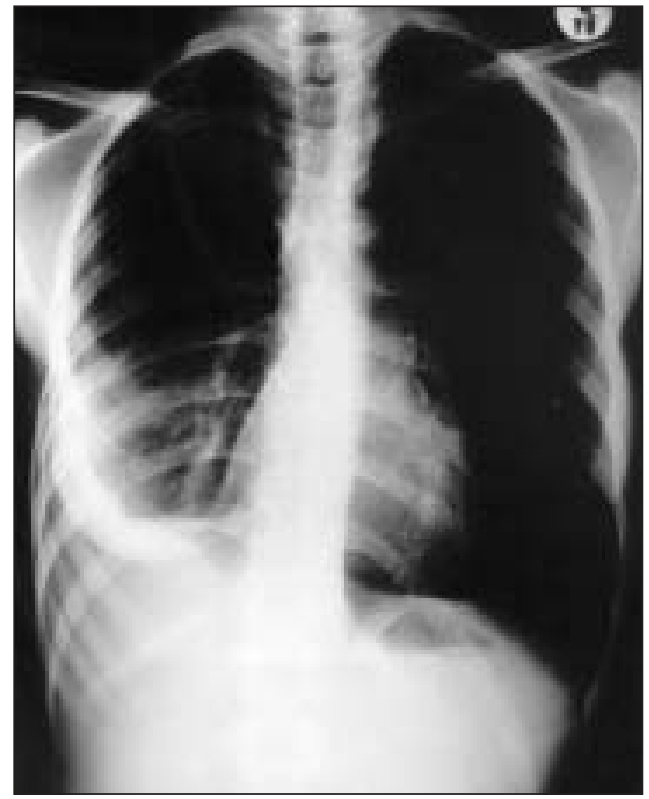
A. Alacacioğlu
A. Akkoçlu ${ }^{2}$
F. Fidan ${ }^{2}$
N. Dutar ${ }^{2}$
I. Alacacioğlu ${ }^{1}$
T. Canda

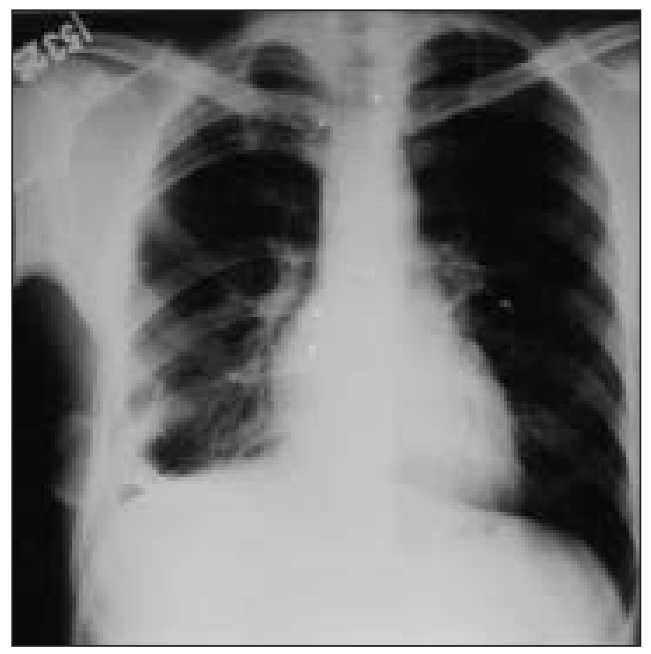

Figure 1

Chest radiographs at admission: a) posterior-anterior; and b) lateral decubitus.

\section{Task 1}

Interpret the chest radiograph. 


\section{Answer 1}

Chest radiography revealed a right hemithorax retraction and blunting of the right costophrenic angle.

In addition, thoracic computed tomography (CT) was performed and is shown on figure 2 .

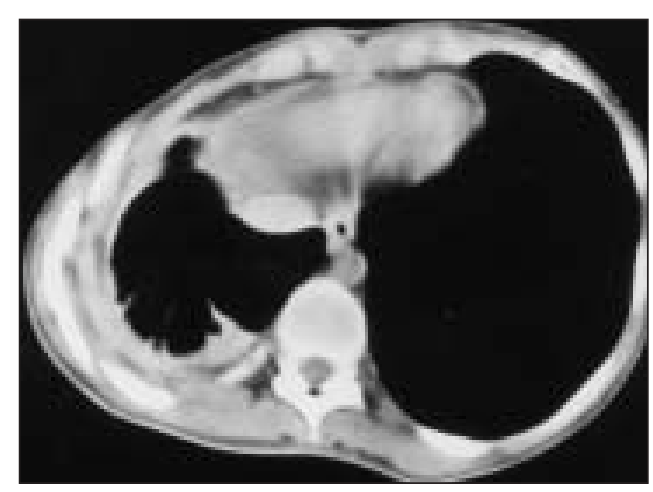

Figure 2

A thoracic CT at admission.

\section{Task 2}

Interpret the CT scan.

\section{Answer 2}

Thoracic CT showed volume loss of right hemithorax, and pleural thickening of the inferior and middle zone of the right lung.

Thorax ultrasonography revealed a right minimal effusion, and pulmonary function tests showed a restrictive pattern. On fibreoptic bronchoscopy, there was no endobronchial lesion. There were no acidoresistant bacilli in bronchoalveolar lavage fluid (BAL) and cultures were also negative. Cytology of BAL showed $95 \%$ polymorphonuclear leukocytes and 2\% lymphocytes. Later on, an open pleural biopsy was performed and a histopathological analysis was carried out, the results of which are shown in figure 3 .

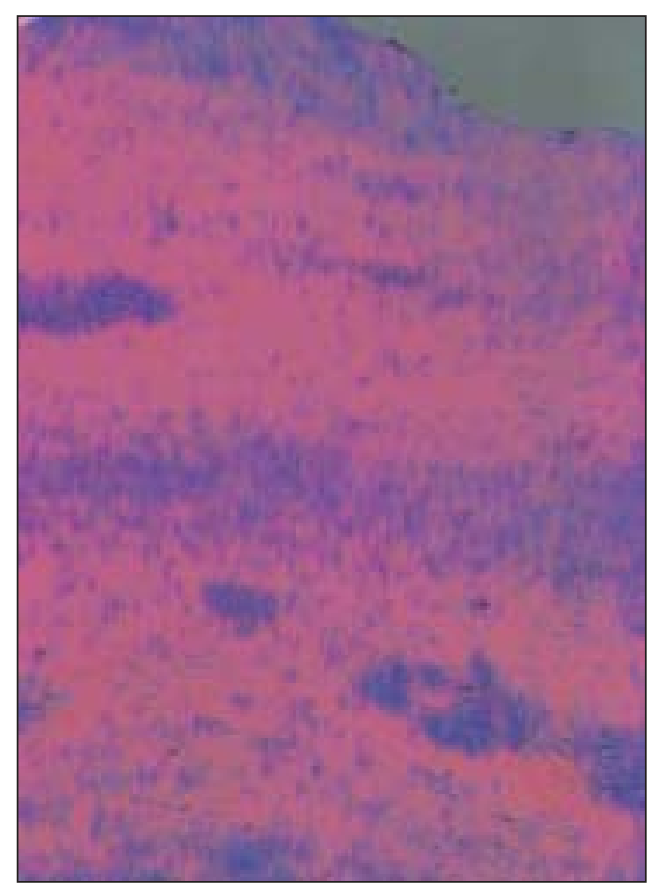

Figure 3

Histopathological findings.

\section{Task 3.}

Interpret the histopathological findings.

Task 4.

Suggest a diagnosis. 


\section{Answer 3}

Histopathological examination demonstrated chronic non-specific inflammation.

\section{Answer 4}

Pleural thickening secondary to bromocriptine treatment.

\section{Discussion}

Bromocriptine is an ergot derivative with dopaminergic activity. It is used in the treatment of Parkinson's disease and hyperprolactinemia. Higher doses are required to cross the blood-brain barrier in these diseases. There are some reported side-effects of this drug, such as headache, nausea and orthostatic hypotension. Rarely, pleural effusion and pleural thickening have been reported as a result of long-term and high-dose treatment, but others have denied a causal relationship [1-13].

Pleuropulmonary changes during bromocriptine treatment have been reported by Rinne [9], who reviewed the charts of 123 patients with Parkinson's disease receiving long-term bromocriptine treatment. Seven of these patients developed pleuropulmonary disease, which included pleural effusions, pleural thickening and pulmonary infiltrates. The patients received bromocriptine therapy alone or in association with levodopa at doses of 20-90 mg daily for 6-27 months prior to the development of symptoms. No specific cause was determined for the pleuropulmonary changes and, with the withdrawal of bromocriptine, clinical and radiological improvement were observed in two patients.

Tornling et al. [11] reported four cases of pleuropulmonary disease during treatment with bromocriptine at doses of $20-50 \mathrm{mg}^{-}$day ${ }^{-1}$. Three patients who received $\geq 50 \mathrm{mg} \cdot$ day $^{-1}$ of bromocriptine developed respiratory symptoms, an elevated ESR, pulmonary infiltrates and pleural fibrosis with associated effusions, with partial resolution following withdrawal of the drug or reduction in dosage. However, pleural changes were not completely reversible in all patients.

Kinnunen and Viljanen [5], McElvaney et al. [8], Vergeret [14], Wiggins and Skinner [15], and Le Witt and Calne [7] also described 14 cases with bromocriptine-dependent pleuropulmonary changes. The patients had Parkinson's disease and were all treated with bromocriptine in a dosage range of 22-100 mg.day ${ }^{-1}$. Physicians observed pleuropulmonary changes 9-48 months after the beginning of treatment. They reported clinical and radiological improvement after the discontinuation of bromocriptine treatment, but progression in the cases of continuation of therapy at 2 years of follow-up.

Morelock and Sahn [16] reported more than 20 cases of pleuropulmonary disease attributed to bromocriptine in the period 1966-1998 in the literature. Radiographical changes reported in these patients included pleural effusion, pleural thickening and interstitial infiltrates. The onset occurred 12-48 months after institution of therapy.

While pleural fluid eosinophilia (12-30\%) has been noted in two patients [8], the majority of pleural fluid analysis has revealed a lymphocytic predominant exudate (51-99\%) without eosinophilia. Drug therapy withdrawal leads to resolution of pleural effusions, but pleural thickening and interstitial parenchymal changes do not resolve completely in all patients.

McElvaney et al. [8] have previously reported 23 patients receiving bromocriptine therapy who developed pleuropulmonary disease. All were male and the majority had a history of long-term cigarette smoking. The authors also proposed that age could be a risk factor, since the majority of cases have been reported in patients $>60$ years, but this may be also related to the age at which Parkinson's disease occurs.

The mechanism of bromocriptine-induced pleuropulmonary disease is unclear, but is probably an idiosyncratic or hypersensitivity reaction. Similar pleuropulmonary changes can occur with other dopamine agonist ergot derivatives (ergotamine), because their molecular structures and pharmacological properties are comparable. Furthermore, bromocriptine, methysergide and ergotamine have all been associated with the development of retroperitoneal fibrosis [8].

Pleuropulmonary changes have been observed with high doses of bromocriptine treatment and long-term usage. Thus, the cumulative dose of bromocriptine may be responsible for these effects [2]. In some patients, radiological and clinical improvement have been observed after the discontinuation of bromocriptine. Prednisolone therapy has been used in some patients, but usefulness of prednisolone is not established [13].

The patient presented here received bromocriptine at a dose of $60 \mathrm{mg} \cdot$ day $^{-1}$ for 10 months. There was no other identified cause of pleural thickening. The open pleural biopsy specimen displayed chronic inflammation. It was 
accepted that all of these findings were dependent on bromocriptine and the therapy was stopped. Subsequently, steroid treatment was initiated. The symptoms of the patient consequently regressed. She was discharged and followed in the outpatient clinic for radiological remission.

\section{References}

1. Melmed S, Braunstein GD. Bromocriptine and pleuropulmonary disease. Arch Intern Med 1989; 149: 258-259.

2. Bhatt MK, Kenan SP, Fleetham JA, et al. Pleuropulmonary disease associated with dopamine agonist therapy. Ann Neurol 1991; 30: 613-616.

3. Hely MA, Morris JG, Lawrence $S$, et al. Retroperitoneal fibrosis, skin and pleuropulmonary changes associated with bromocriptine therapy. Aust N Z J Med 1991; 21: 82-84.

4. Hillerdal $G$, Lee J, Blokust $A$, et al. Pleural disease during treatment with bromocriptine in patients previously exposed to asbestos. Eur Respir J 1997; 10: 2711-2715.

5. Kinnunen E, Viljanen A. Pleuropulmonary involvement during bromocriptine treatment. Chest 1988; 94: 1034-1036.

6. Knoop C, Mairesse M, Lenclud C, et al. Pleural effusion during bromocriptine exposure in two patients with pre-existing asbestos pleural plaques: a relationship? Eur Respir J 1997; 10: 2898-2901.

7. Le Witt $P A$, Calne DB. Pleuropulmonary changes during long-term bromocriptine treatment of Parkinson's disease. Lancet 1981; 1: 44-45.

8. McElvaney $N G$, Wilcox $P G$, Churg $A$, et al.
In conclusion, bromocriptine-dependent pleural thickening is rare and other usual causes of pleural diseases should be first excluded. However, physicians should remember that pleuropulmonary changes can occur in high-dosage bromocriptine therapy.

Pleuropulmonary disease during bromocriptine treatment of Parkinson's disease. Arch Intern Med 1998; 148: 2231-2236.

9. Rinne UK. Pleuropulmonary changes during long-term bromocriptine treatment for Parkinson's disease. Lancet 1981; 1: 44.

10. Todman DH, Oliver WA, Edwards RL. Pleuropulmonary fibrosis due to bromocriptine treatment for Parkinson's disease. Clin Exp Neurol 1990; 27: 79-82.

11. Tornling G, Unge G, Axelsson G, Noring L, Granerus AK. Pleuropulmonary reactions in patients on bromocriptine treatment. Eur J Respir Dis 1986; 68: 35-38.

12. Ward CD, Thompson J, Humby MD. Pleuropulmonary and retroperitoneal fibrosis associated with bromocriptinetreatment. J Neurol Neurosurg Psychiatry 1987; 50: 1706-1707.

13. Weil $C$. The safety of bromocriptine in long term use: a review of the literature. Curr Med Res Opin 1986; 10: 25-51.

14. Vergeret J, Barat M, Taytard A, et al. Fibrose pleuro pulmonaire et bromocriptine. Sem Hop Paris 1984; 60: 741-744.

15. Wiggins J, Skinner C. Bromocriptine induced pleuropulmonary fibrosis. Thorax 1986; 41: 328-330.

16. Morelock SY, Sahn SA. Drug and pleura. Chest 1999; 116: 212-221. 